\title{
Session X
}

\section{NuCleosynthesis AND Evolution}




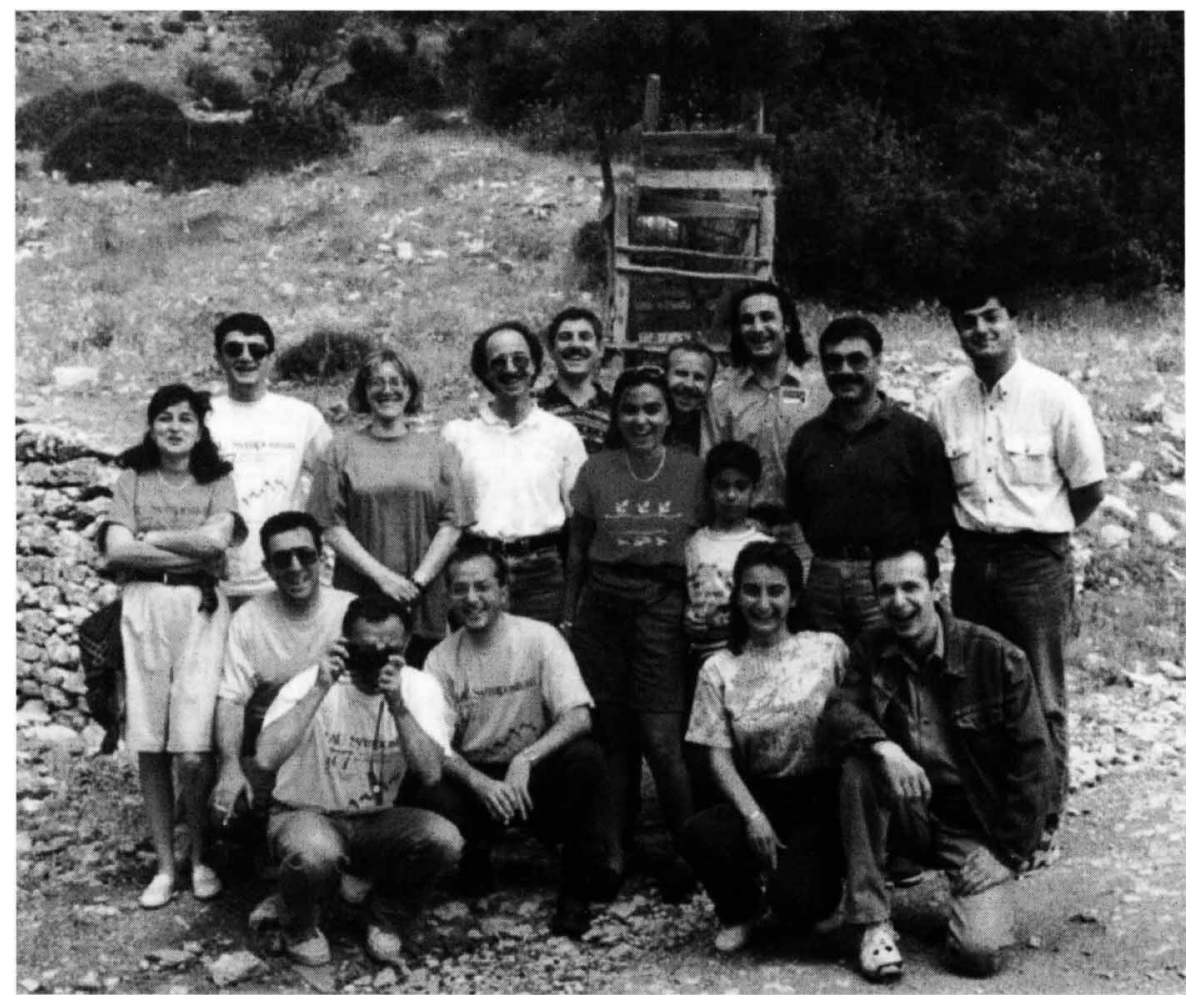

Turkish students and their partners gather for a photo on the way to Bakırlitepe, while the bus driver adds cool spring water to his radiator. 


\title{
HEAVY-ELEMENT NUCLEOSYNTHESIS IN AGB STARS
}

\author{
VERNE V. SMITH \\ Dept. of Physics, University of Texas at El Paso \\ El Paso, TX 79968, U.S.A. \\ and \\ McDonald Observatory, University of Texas at Austin \\ Austin, TX 78712, U.S.A.
}

\begin{abstract}
The production of certain neutron-rich elements heavier than iron occurs during He shell-burning on the asymptotic giant branch (AGB). These neutron captures occur at rather low neutron densities and, thus, the resulting heavy-element nucleosynthesis is characterisitic of the so-called $s$ process. Abundance analyses of the $s$-process elements in AGB stars can reveal details of the neutron densities and the stage of AGB evolution at which $s$-processing occurs, as well as the nature of the neutron source. These details derived from observations can constrain models of stars evolving along the AGB. Recent results concerning the nature of the $s$-process as a function of metallicity and the nature of the neutron source are reviewed.
\end{abstract}

\section{Introduction}

Many of the types of stars discussed at this symposium on "The Carbon Star Phenomenon" have played a key role in our understanding of the origins of the chemical elements. The idea that many (and as it has turned out, most) of the elements heavier than helium can be synthesized in the interiors of stars certainly received strong affirmation by Merrill's (1952) classic, and oft cited, spectroscopic detection of Tc I in certain S stars. With only relatively short-lived isotopes (half-lives of $\sim 10^{5}-10^{6} \mathrm{yrs}$ ), the observed technetium is "currently" synthesized in the interiors of these red giants and mixed to their surfaces. The production of Tc, and most of the other heavy elements (i.e. those heavier than $\mathrm{Fe}$ ), is driven by successive neutron captures and $\beta$-decays which build up the range of elements beyond iron. Two neutron-capture processes are recognized: one happens at rapid capture rates which exceed the $\beta$-decay rates, and is referred to as the 
$r$-process. The other process occurs at capture rates which are less than typical $\beta$-decay rates and is referred to as the $s$-process. The details of the $s$ - and $r$-processes were elucidated in the classic work by Burbidge, Burbidge, Fowler \& Hoyle (1957). The technetium observed by Merrill in the $\mathrm{S}$ stars was created at low neutron-capture rates (low neutron densities) and is a product of the $s$-process.

It has been known since the work of Schwarzschild \& Harm (1967) and Sanders (1967) that the preferred astrophysical site for the production of much of the $s$-process nuclei was a region associated with the $\mathrm{H}$ - and ${ }^{4} \mathrm{He}-$ burning shells found in stars evolving along the asymptotic giant branch (AGB). Of course, it is in these same ${ }^{4} \mathrm{He}$-burning shells that ${ }^{12} \mathrm{C}$ is made, which is then mixed to the surface to produce the C-rich stars we are discussing at this meeting: the $s$-process heavy elements and ${ }^{12} \mathrm{C}$ are thus intertwined. Much of the early work connecting stellar models evolving through the AGB to $s$-process nucleosynthesis was carried out by Iben and collaborators and is summarized nicely in the review by Iben \& Renzini (1983).

This is a brief discussion of a few current topics concerning the $s$-process in AGB stars, but we list here for the reader some other recent reviews and papers which cover various aspects of this topic: Smith (1990) and Lambert (1991) cover observational topics, while Iben \& Renzini (1983) and Lattanzio (1989) present excellent overall reviews of theory.

\section{The Heavy-Element Rich AGB Stars}

As quasi-periodic shell ${ }^{4} \mathrm{He}$-burning occurs during thermal pulses on the $\mathrm{AGB}$, mixing episodes increase the atmospheric $\mathrm{C} / \mathrm{O}$ ratio in these red giants, giving rise to the spectral sequence of $\mathrm{M}$ to $\mathrm{MS}$ to $\mathrm{S}$ to $\mathrm{C}$. This increase in the $\mathrm{C} / \mathrm{O}$ is accompanied, in general, by increasing $s$-process overabundances, so that the MS, S, and C stars are also heavy-element rich AGB stars. In addition, there are a number of warmer, lower-luminosity stars which exhibit $s$-process enhancements, such as the $\mathrm{CH}$ and barium stars (Keenan 1942; Bidelman \& Keenan 1951). As first demonstrated convincingly by McClure, Fletcher \& Nemec (1980) and most recently by McClure \& Woodsworth (1990), these lower-luminosity heavy-element rich stars are members of binary systems whose companions, now white dwarfs, transferred heavy elements when they were AGB stars.

A number of detailed abundance analyses have been conducted on the various heavy-element rich stars over the last 15 years and can be summarized as follows: for the barium stars we have Tomkin \& Lambert (1983, 1986), Smith (1984), Kovacs (1985), Smith \& Lambert (1984), Malaney \& Lambert (1988), and Busso et al. (1995). The CH stars have been studied 
by Vanture (1992a, 1992b). The MS and S stars have been studied by Smith \& Lambert (1990), Plez, Smith \& Lambert (1993), Busso et al. (1995), and Lambert et al. (1995), while the most detailed analysis of the $s$-process in the carbon stars is still the work of Utsumi (1985). Recently, Smith et al. $(1996,1997)$ have identified the yellow symbiotic binary systems AG Dra and $\mathrm{BD}-21^{\circ} 3873$ as metal-poor members of the Ba star class.

Taken together, these abundance studies provide the observational results which can be used to probe our current understanding of the details of $s$-process nucleosynthesis in AGB stars and we now summarize these results.

\section{The $s$-Process as a Function of Metallicity}

One question that is basic to an understanding of the $s$-process in AGB stars is the nature of the neutron source. Since the realization that the slow capture of neutrons is a distinct stellar nuclear process, two candidate neutron producing reactions have been suggested: ${ }^{13} \mathrm{C}(\alpha, \mathrm{n}){ }^{16} \mathrm{O}$ and ${ }^{22} \mathrm{Ne}(\alpha, \mathrm{n}){ }^{25} \mathrm{Mg}$ (Cameron 1955; Greenstein 1954). These two separate sources have very different characters; the $s$-process in AGB stars is associated with ${ }^{4} \mathrm{He}-$ burning temperatures of $\mathrm{T} \geq 10^{8} \mathrm{~K}$, with the ${ }^{13} \mathrm{C}$ source being active near $1 \times 10^{8} \mathrm{~K}$, while the ${ }^{22} \mathrm{Ne}$ source requires higher temperatures, nearer to $2-3$ $\times 10^{8} \mathrm{~K}$. The different temperatures required to drive the $s$-process via the different neutron sources will occur in AGB stars of different mass, or at different points along the AGB. Historically, the ${ }^{22} \mathrm{Ne}$ source was favored as the most likely $s$-process candidate because the production of ${ }^{22} \mathrm{Ne}$ can occur quite naturally as the result of succesive $\alpha$-captures onto ${ }^{14} \mathrm{~N}$ in the He-burning shell of an AGB star $\left({ }^{14} \mathrm{~N}(\alpha, \gamma){ }^{18} \mathrm{~F}\left(\mathrm{e}^{+}, \nu\right){ }^{18} \mathrm{O}(\alpha, \gamma){ }^{22} \mathrm{Ne}\right)$, with the initial ${ }^{14} \mathrm{~N}$ itself coming from CNO-cycle H-burning. The ${ }^{13} \mathrm{C}$ neutron source required an extra mixing mechanism to move protons into the $\mathrm{He}-$ burning shell to produce ${ }^{13} \mathrm{C}$ via ${ }^{12} \mathrm{C}(\mathrm{p}, \gamma){ }^{13} \mathrm{~N}\left(\beta^{+}, \nu\right){ }^{13} \mathrm{C}$, and the physical nature of this extra mixing was not clear. The physics involved in any sort of extra mixing is both complicated and not easily constrained by modelling alone. However, the expected behavior of these neutron sources with metallicity is different and can be tested by observation. If the observations can determine the dominant neutron source, this alone can provide important constraints for stellar models.

A convenient parameter to characterize the relative abundance distribution of the $s$-process is the neutron exposure, $\tau$, defined as

$$
\tau=\int_{0}^{t^{\prime}} N_{n}(t) V(t) d t
$$

where $N_{n}$ is the neutron density and $V(t)$ the relative velocity of neutrons and nuclei, with the integral taken over the interval of the $s$-process 
episode. A large neutron exposure leads to a larger amount of heavier nuclei produced (e.g. $\mathrm{Ba}, \mathrm{La}$, or $\mathrm{Ce}$ ) relative to the lighter nuclei, such as $\mathrm{Y}$ or $\mathrm{Zr}$.

As pointed out by Clayton (1988), the nature of the dominant $s$-process neutron source can be tested by using the heavy- to light-element abundance distribution of the $s$-process (a measure of $\tau$ ) as a function of metallicity. For example if, during the $s$-process episode, the neutrons are in local equilibrium between their production and destruction, then the neutron density will be

$$
N_{n}=\left(\Sigma N_{i} N_{j}\langle\sigma\rangle_{i, j}\right) /\left(\Sigma N_{k}\langle\sigma\rangle_{k, n}\right)
$$

where the numerator is summed over all neutron-producing reactions between species $i, j$, with Maxwellian-averaged cross sections of $\langle\sigma\rangle_{i, j}$, and the denominator has the neutron destruction (absorbing) reactions, summed over all neutron absorbers $k$, and again a Maxwellian-averaged neutronabsorption cross section of $\langle\sigma\rangle_{k, n}$. Making the simplifying assumption that the neutron-producing reactions come from $\alpha$-captures, and that the dominant neutron absorbers are the seed nuclei of $\mathrm{Fe}$, the above expression can be approximated as

$$
N_{n} \propto\left(N_{H e} N_{j}\right) /\left(N_{F e}\right),
$$

where $N_{j}=N\left({ }^{13} \mathrm{C}\right)$ for the ${ }^{13} \mathrm{C}$ source and $N\left({ }^{22} \mathrm{Ne}\right)$ for the ${ }^{22} \mathrm{Ne}$ source. Since the ${ }^{22} \mathrm{Ne}$ in the He-burning layers comes from ${ }^{14} \mathrm{~N}$ which, itself, comes from the initial $\Sigma \mathrm{CNO}$ which is $\propto N_{F e}$, the expected neutron density (and neutron exposure) would be expected to be roughly independent of metallicity. ${ }^{13} \mathrm{C}$, on the other hand, must result from the mixing of protons into a ${ }^{12} \mathrm{C}$-rich region, where the ${ }^{12} \mathrm{C}$ comes from ${ }^{4} \mathrm{He}$, which is almost independent of metallicity. Thus, if the structures of the He-shells in AGB stars are not strong functions of metallicity, the numerator of the above expression is constant and $N_{n}$ (and $\tau$ ) is $\propto N_{F e}{ }^{-1}$.

The neutron exposure can be estimated using published abundance results and we show the results of such an exercise in Figure 1 on the next page. Here we have plotted the quantity $[\mathrm{hs} / \mathrm{ls}]$ vs. $[\mathrm{Fe} / \mathrm{H}]$ from a number of studies. The quantity [hs/ls] represents the heavy- $s$ to light- $s$ ratio and is an average overabundance of representative heavy $s$-process nuclei $(\mathrm{Ba}$, $\mathrm{La}$, and $\mathrm{Ce}$ ) relative to an average overabundance of the light $s$-process species $\mathrm{Y}$ and $\mathrm{Zr}$, measured in standard spectroscopic bracket notation. This ratio [hs/ls] is proportional to the neutron exposure defining the $s$ process abundance distribution. The scatter is rather large, although this is not surprising as we are comparing results from a large number of investigators using somewhat different techniques and data of differing quality. Nonetheless, there is a clear and significant trend of increasing [hs/ls] with decreasing metallicity. That is, there is a significant increase of neutron exposure with decreasing metallicity as predicted from Clayton's (1988) 


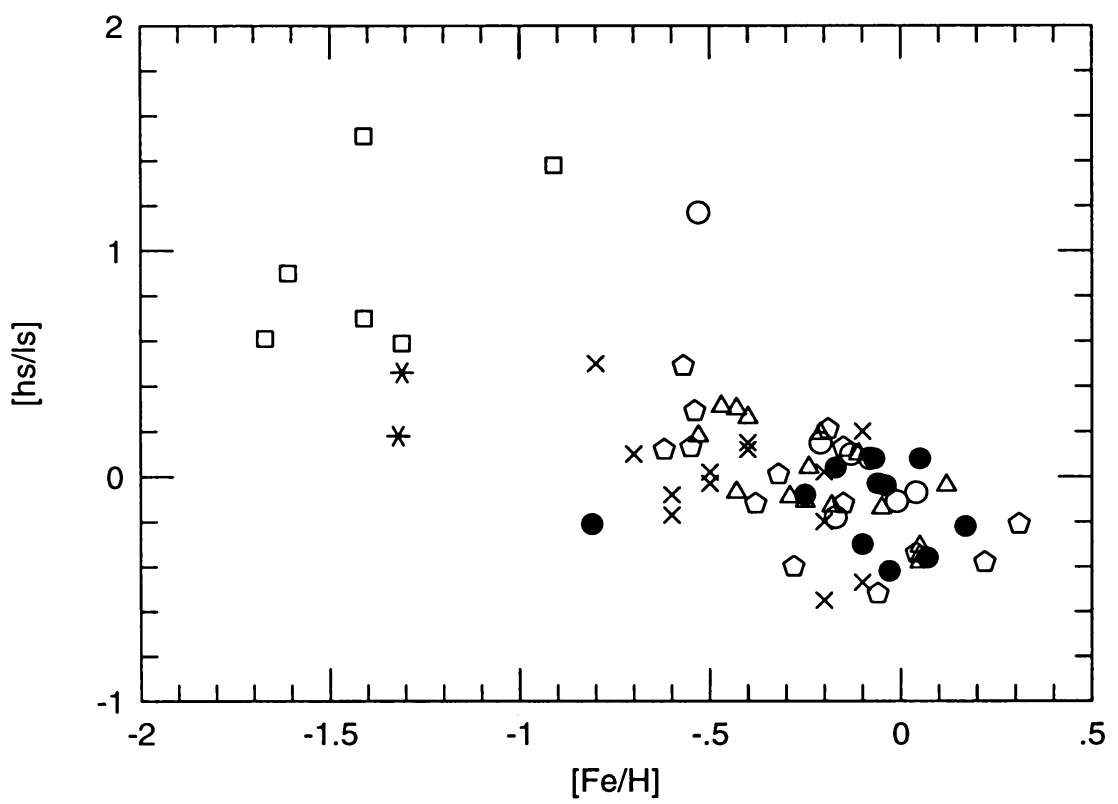

Figure 1. Published abundance results for the heavy $s$-process elements (Ba, La, and $\mathrm{Ce}$ ) relative to the light $s$-process species ( $\mathrm{Y}$ and $\mathrm{Zr}$ ) for the MS/S stars (filled circles, with Tc, and open circles, without Tc), Ba giants (open triangles), Ba dwarfs (open pentagons), $\mathrm{CH}$ giants (open squares), $\mathrm{CH}$ subgiants (crosses), and yellow symbiotics (six-pointed stars). The increase of [hs/ls], which indicates an increasing neutron exposure, with decreasing metallicity $[\mathrm{Fe} / \mathrm{H}]$ is evident. Such an increase is predicted by the ${ }^{13} \mathrm{C}(\alpha, \mathrm{n}){ }^{16} \mathrm{O}$ neutron source.

simple argument for the ${ }^{13} \mathrm{C}(\alpha, \mathrm{n})$ neutron source. Based on the observations, it would appear that the current best candidate for the $s$-process neutron source in AGB stars is ${ }^{13} \mathrm{C}$.

\section{Summary}

The topic of heavy-element nucleosynthesis in AGB stars is an exciting one. There is now emerging a unified picture which identifies the $\mathrm{N}$-type carbon stars and the MS/S stars with technetium as AGB stars currently in the throes of active dredge-up, while the idea of mass transfer in a wide binary system adds the interpretation of dwarf, subgiant, and giant $\mathrm{Ba}$ and $\mathrm{CH}$ star abundance peculiarities as due to material processed in an AGB star being added to an "innocent companion." There is a wealth of observational and theoretical data being obtained concerning nucleosynthesis in AGB stars and it is fair to say that we are experiencing a real convergence of theory and observation which is leading us toward a much deeper understanding of this phase of stellar evolution. 


\section{References}

Bidelman, W.P. \& Keenan, P. C. 1951, ApJ, 114, 473

Burbidge, E. M., Burbidge, G. R., Fowler, W. A. \& Hoyle, F. 1957, Rev. Mod. Phys., 29, 4 Busso, M., Gallino, R., Lambert, D. L., Raiteri, C. M. \& Smith, V. V. 1992, ApJ, 399, 218

Busso, M., Lambert, D.L., Beglio, L., Gallino, R., Raiteri, C. M. \& Smith, V. V. 1995, $A p J, 446,775$

Cameron, A. G. W. 1955, ApJ, 121, 144

Clayton, D. D. 1988, MNRAS, 234, 1

Gonzalez, G. \& Wallerstein, G. 1994, $A J, 108,1325$

Greenstein, J. L. 1954, in Modern Physics for Engineers, ed. J. L. Ridenour (McGrawHill), p. 99

Iben, I. Jr. \& Renzini, A. 1983, Ann. Rev. Astron. Astrophys., 21, 271

Keenan, P. C. $1942, A p J, 96,101$

Kovacs, N. 1985, $A \& A, 150,232$

Lambert, D. L. 1991, in IAU Symp. 145: Evolution of Stars: The Photospheric Abundance Connection, ed. G. Michaud and A. Tutukov (Kluwer), p. 299

Lambert, D. L., Smith, V. V., Busso, M., Gallino, R. \& Straniero, O. 1995, ApJ, 450, 302

Lattanzio, J. 1989, in IAU Coll. 106: Evolution of Peculiar Red Giant Stars, ed. H. R. Johnson and B. Zuckerman (Cambridge Univ. Press), p. 161

Malaney, R. A. \& Lambert, D. L. 1988, MNRAS, 235, 695

McClure, R. D., Fletcher, J. M. \& Nemec, J. M. 1980, ApJ, 238, L35

McClure, R. D. \& Woodsworth, A.W. 1990, ApJ, 352, 709

Merrill, P. W. 1952, ApJ, 116, 21

Norris, J. E. \& Da Costa, G.S. 1995, $A p J, 447,680$

Plez, B., Smith, V. V. \& Lambert, D. L. 1993, ApJ, 418, 812

Sanders, R. H. 1967, ApJ, 150, 971

Schwarzschild, M. \& Harm, R. 1967, ApJ, 150, 961

Smith, V. V. 1984, $A \& A, 132,326$

Smith, V. V. 1990, in Cool Stars, Stellar Systems and the Sun, ed. G. Wallerstein, ASP Conf. Ser., 9, 340

Smith, V. V., Cunha, K., Jorissen, A. \& Boffin, H. M. J. 1996, $A \& A, 315,179$

Smith, V. V., Cunha, K., Jorissen, A. \& Boffin, H. M. J. 1997, $A \& A, 324,97$

Smith, V. V. \& Lambert, D. L. 1984, PASP, 96, 226

Smith, V. V. \& Lambert, D. L. 1990, ApJ Supp., 72, 387

Tomkin, J. \& Lambert, D. L. 1983, $A p J, 273,722$

Tomkin, J. \& Lambert, D. L. 1986, ApJ, 311, 819

Utsumi, K. 1985, in Cool Stars with Excesses of Heavy Elements, ed. M. Jaschek and P. C. Keenan (Kluwer), p. 243

Vanture, A. D. $1992 \mathrm{a}, A J, 103,2035$

Vanture, A. D. 1992b, $A J, 104,1997$

Vanture, A. D., Wallerstein, G. \& Brown, J. A. 1994, PASP, 106, 835

\section{Discussion}

Plez: Our observations of the $\mathrm{Rb} / \mathrm{Zr}$ ratio in high-luminosity AGB stars in the SMC (Plez, Smith \& Lambert 1993, ApJ, 418, 812) also fall on the general trend on $\mathrm{Rb} / \mathrm{Zr}$ vs. $\mathrm{Fe} / \mathrm{H}$ that you have shown.

Smith: Yes, and these SMC stars are some of the more luminous and, thus, fairly massive AGB stars. 